\title{
Disruptive MEMS Technology Replaces Conventional Bead Pellistor Device
}

\author{
Stephan Trautweiler ${ }^{1}$, Nicolas Moser ${ }^{1}$, Hervé Delprat ${ }^{1}$, Sophie Lepoutre ${ }^{1}$, Christine Alépée ${ }^{1}$, Terry \\ Brown ${ }^{2}$, Kevin Brown ${ }^{2}$ \\ ${ }^{1}$ e2v microsensors SA, Rue des Courtils 1, 2035 Corcelles - Switzerland, \\ strautweiler@microchemical.com \\ ${ }^{2}$ e2v technologies, 106 Waterhouse Lane, Chelmsford, Essex, CM1 2QU - United Kingdom
}

\begin{abstract}
:
An industrial-suited MEMS (Micro Electro Mechanical System) is developed to launch a new generation of high-volume, low-cost and rugged sensors for the detection of combustible gases, in particular methane. Innovative process technologies combined with optimized sensing and filter materials are engineered to achieve equal or exceeding performance compared to existing products. At the same time manufacturing cost is significantly reduced. This paper outlines the concept and features of our new MEMS device and demonstrates its benefits by presenting industrial-type test methods and measurement results.
\end{abstract}

Key words: MEMS, high-volume, low-cost, methane, filter, ATEX

\section{Motivation - The Needs for Innovative Solutions}

Methane explosions in coalmines have caused tens of thousands of deaths throughout the history of mining. The worst Chinese disaster in 1942 killed more than 1500 people. In 1963 a methane explosion in Japan and a concomitant carbon monoxide buildup claimed the lives of over 450 miners. Nowadays mine workers are equipped with portable detectors to warn them from the presence of dangerous methane levels. The most commonly used sensing device used in these detectors is the catalytic pellistor. It consists of a coiled platinum wire covered by a catalyst-doped ceramic coating. The platinum wire serves as a heating resistor and the ceramic coating usually forms a beadshaped pellet, hence the name "Pellet-Resistor" or "Pellistor" [1].

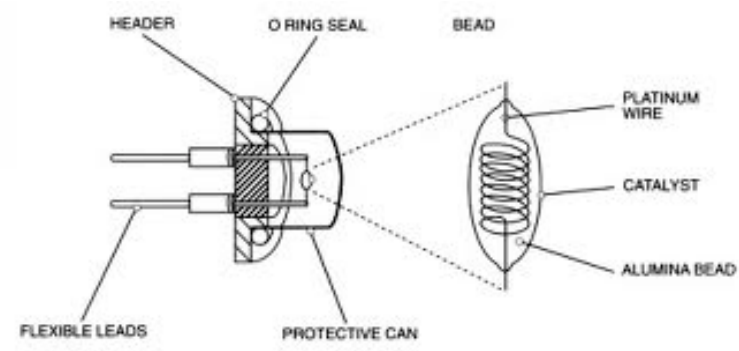

Fig. 1. Cross-section of a pellistor device showing the enlarged sensing bead.

The working principle is schematically described in Figure 2. Two pellistors, one called
Detector (doped with catalyst) and one called Compensator (without catalyst) are connected electrically with two paired resistors to form a Wheatstone bridge circuit.

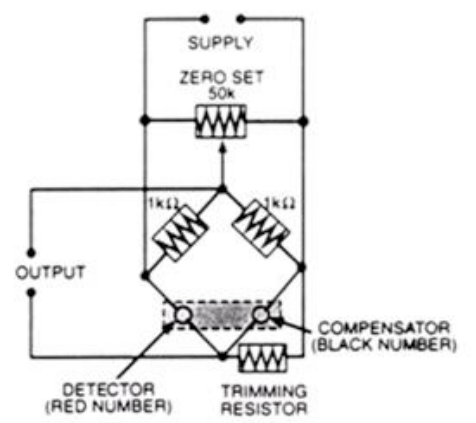

Fig. 2. Wheatstone bridge measurement circuit with catalyst-doped pellistor for gas detection (Detector) and matched pellistor without catalyst for temperature compensation (Compensator).

Applying a supply voltage forces a current to flow through both platinum coils and heats the beads up to their operating temperature of around $500^{\circ} \mathrm{C}$. As the resistance of the platinum wire changes with temperature it also acts as a calorimeter. The porous alumina bead offers large reaction surface and uniform temperature distribution due to its high thermal conductivity. In the absence of combustible gases, both Detector and Compensator resistance values vary by the same amount when the device is turned on or when ambient temperature changes. The Wheatstone bridge remains 
balanced and the output voltage equals zero. When methane is present in the ambient it is oxidized on the catalytic surface of the Detector to form carbon dioxide and water. The released reaction heat produces a temperature rise and consequently an additional resistance increase in the Detector which unbalances the Wheatstone bridge. As a result the output voltage increases proportionally with the concentration of methane.

Pellistors have been in use for the detection of combustible gases for more than 50 years. The fabrication process is relatively expensive as it involves delicate operation steps such as wire coiling, electric connection and ceramic coating. Typical wire diameters are only about $20 \mu \mathrm{m}$ as shown in Fig. 3. Spread in resistance values are unavoidable and labor-intensive pairing of matching resistors is required, adding further cost to the process. Low power consumption a key parameter for portable instruments - is achieved by suspending the wires between electric contact posts [2]. Shocks or vibrations can cause the suspended platinum wires to brake and destroy the device. In order to make combustible gas sensing more affordable and robust, the industry is in strong need for technologic breakthroughs.

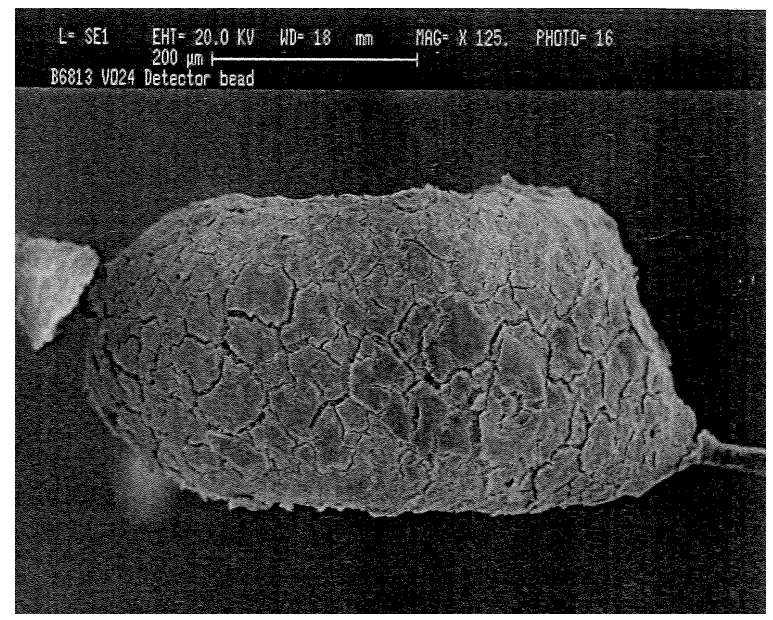

Fig. 3. Scanning electron micrograph of a pellistor bead measuring approximately 500 $\mu \mathrm{m}$ in length.

\section{Design - Benefits of MEMS Technology}

MEMS are ideally suited for high-volume, lowpower, and mechanically robust applications [3]. The device discussed in this article is a $3 \mathrm{~mm} \times 3 \mathrm{~mm} \times 0.4 \mathrm{~mm}$ silicon chip with a thermally isolating membrane obtained by etching a cavity in $\mathrm{KOH}$ from the backside of the chip. The resulting structure provides the mechanical support for the heating resistor and the sensitive layer. Instead of a 3-dimensional coil, a 2-dimensional meander is formed on top of the membrane. The original catalyst-doped alumina bead is replaced by a layer deposited over the heating meander.

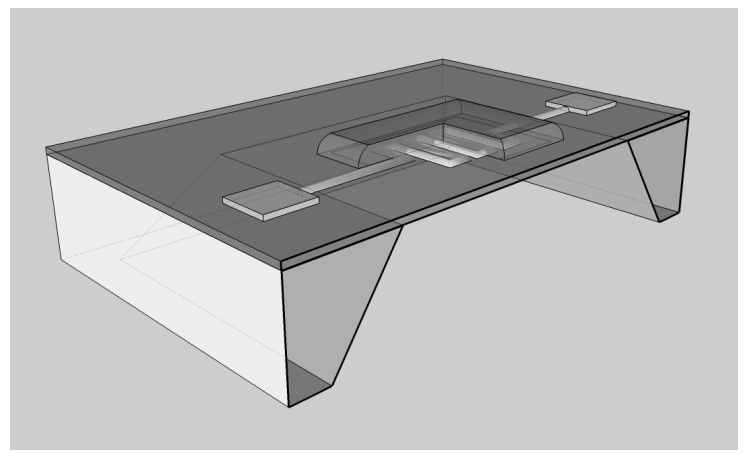

Fig. 4. Micromachined silicon chip with thermally isolating membrane, electric heating resistor and catalytic sensing layer.

Two major improvements are thereby achieved; (one) mechanical robustness to vibrations and shocks and (two) reduced power consumption. Determinant for the mechanical strength " $\sigma$ " are the mass " $m$ " of the moving part subjected to acceleration "a" (i.e. the bead respectively the sensing layer) and the characteristic crosssection "A" (i.e. the cross-section of the wire respectively the membrane) onto which the acceleration force " $\mathrm{F}_{\mathrm{acc}}$ " is exerted according to eq. (1).

$\sigma=\frac{F_{a c c}}{A}=\frac{m \times a}{A}$

Considering a ratio of masses between the bead and the sensing layer of 15 and a ratio of characteristic surface areas of 0.15 the resulting tensile stress caused by the same acceleration (vibration or shock) is 100 times less in the case of our MEMS structure. Furthermore, taking into account that the maximum tensile strength of the dielectric material used for the membrane (>1000 MPa) is more than 5 times higher than the one for the platinum wire (200 MPa) a global increase in mechanical robustness by a factor of at least 500 is achieved. Power consumption depends on heat losses which are dominated by convection with the ambient air and conduction through the metal wires. The electric power required to bring the device to its operating temperature is described in the eq. (2).

$P_{e l}=P_{c o n v}+P_{c o n d}=h \times A_{s} \Delta T+k \times \frac{A_{c}}{L} \Delta T$

Conductive heat loss is of the order of $1 \mathrm{~mW}$ and therefore it is concluded that the major part of heat is transferred to the ambient by convection. By reducing the surface area of the sensing layer and optimizing the heater design, we improved the electric power consumption from approximately $150 \mathrm{~mW}$ per bead down to 
$60 \mathrm{~mW}$ on the MEMS while maintaining the same sensitivity. The resulting benefit is an increased battery lifetime by more than $50 \%$.

Sensitivity, selectivity and stability are the three most critical design parameters for the sensing layer. The gas concentration range of interest for methane detection in coalmines is $0 . .4 \%$ volume which covers the span from clean air to the lower explosive limit (LEL) of methane. Pellistors exhibit typical sensitivities of $10 \mathrm{mV}$ per $\mathrm{V}$ supply voltage and per $\%$ vol. of methane with good linearity up to $3 \%$. Response times below 20 seconds must be achieved to warn users from a potentially dangerous build-up. Water vapor is the most abundant interfering gas in common air and the sensor should not show significant dependence to its varying concentration. Other potentially interfering gases are larger hydrocarbons and nitric oxide which are well discriminated by the sensor. Stability ensures that the sensor performance remains within the specified limits throughout its lifetime. Two aspects are important to validate, (one) the mechanical stability and (two) the chemical stability. Mechanical stability of the membrane was briefly discussed above. Adhesion of the sensing layer is another aspect that needs to be examined carefully. Contrary to the bead-type pellistor, the sensing layer of a MEMS device is subjected to significant stress caused by the difference of thermal expansion coefficients between the materials involved. Furthermore, MEMS devices fabricated on silicon wafers need to be separated into chips with a dicing saw. This process also causes strain to the sensing layer as the water jet used for cooling and cleaning passes over it. Chemical stability, on the other hand, is concerned with contaminants that may alter the response to the target gas temporarily or permanently. Two types of contaminants can be distinguished. The first one includes inhibitors which adsorb on the catalytic sites and thereby reduce the number of sites available to the target gas. Most common inhibitors are sulfur compounds such as H2S (hydrogen sulfide) with high affinity to catalysts. The process is usually reversible and the sensor recovers after some time in clean air. The second type of contaminants called poisons implies irreversible processes that permanently damage the sensing layer. A particularly destructive compound is HMDSO (hexamethyldisiloxane) which upon reaching the hot surface of the sensing layer decomposes and forms a compact SiO2 coating. This permanently passivates the layer and renders it inert to the target gas.
ATEX 95(derived from the French "Atmosphère Explosive") is a European directive for equipment and protective systems intended for use in potentially explosive atmospheres. As described above, the sensing layer is operated at temperatures up to $500^{\circ} \mathrm{C}$. This could ignite atmospheres of combustible gases above their LEL. To avoid self-ignition, a special package was designed where gas is only allowed to diffuse into the sensor via a filter of specified porosity. This protection concept is known as 'Flameproof' and must meet the requirements of the relevant standards, EN50018 or the latest standard, EN60079. ATEX certification was obtained for our MEMS device through an accredited institution according to ATEX 95 equipment directive 94/9/EC.

In view of the finite lifetime easy on-site replacement of the sensor device is proposed in the package shown below where the sensor with its housing is mounted on a SIM-card type substrate as used in portable computers and mobile phones (see Fig. 5).

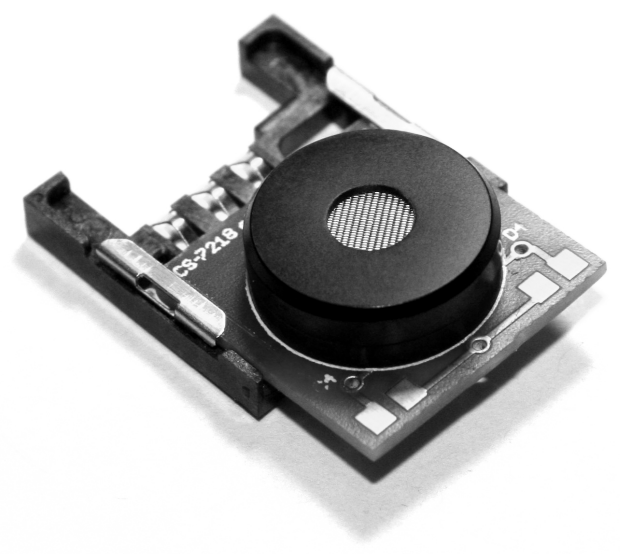

Fig. 5. Packaged MEMS device on a SIM-card $P C B$. The design is suitable for explosion-proof applications (ATEX) and allows easy and quick replacement of the sensing device e.g. when mounted in a portable instrument.

\section{Results - Sensor characteristics under typical test conditions}

The fabricated devices are subjected to an industrial-type series of validation tests to prove their aptitude in the end-use application. Parameters of interest are listed in the table below.

Tab. 1: Parameters and descriptions of validation tests with present status.

\begin{tabular}{|l|l|l|}
\hline Parameter & Test Description & Status \\
\hline Warm-up Time & max. 2 minutes & Passed \\
\hline Peaking Curves & $\begin{array}{l}<2.5 \% \text { variation from } \\
\text { nominal value }\end{array}$ & Ongoing \\
\hline Linearity & $\begin{array}{l} \pm 5 \% \text { LEL or }<10 \% \text { of } \\
\text { indication }\end{array}$ & Passed \\
\hline
\end{tabular}




\begin{tabular}{|c|c|c|}
\hline Response Time & T90<20s & Passed \\
\hline $\begin{array}{l}\text { Short-term } \\
\text { Stability }\end{array}$ & 7 days operation & Passed \\
\hline \begin{tabular}{|l|} 
Vibration \\
\end{tabular} & $2.8 \mathrm{~g}, 10 \ldots 1000 \mathrm{~Hz}$ & Ongoing \\
\hline \begin{tabular}{|l|} 
Drop Test \\
\end{tabular} & $1 \mathrm{~m}$ on concrete floor & Ongoing \\
\hline Orientation & $\begin{array}{l}\text { mounting \& testing in } 6 \\
\text { directions }\end{array}$ & Ongoing \\
\hline \begin{tabular}{|l|} 
Mechanical Shock \\
\end{tabular} & $25 \ldots 100 \mathrm{~g}$ & Ongoing \\
\hline Temperature & $\begin{array}{l}\text { testing at }-10 \ldots 40^{\circ} \mathrm{C} \text { (with } \\
\text { compensation algorithms) }\end{array}$ & Ongoing \\
\hline \begin{tabular}{|l|} 
Pressure \\
\end{tabular} & testing at $80 \ldots 110 \mathrm{kPa}$ & Ongoing \\
\hline Humidity & $\begin{array}{l}\text { testing at } 20 \ldots 90 \% \mathrm{RH} \& \\
40^{\circ} \mathrm{C}\end{array}$ & Ongoing \\
\hline Air Velocity & $0.5 \ldots 3 \mathrm{~m} / \mathrm{s}$ & Ongoing \\
\hline \begin{tabular}{|l} 
Long-term Test \\
\end{tabular} & tested for 3 months & Ongoing \\
\hline \begin{tabular}{|l|} 
High Gas \\
Concentration \\
Exposure
\end{tabular} & $100 \%$ methane & Ongoing \\
\hline \begin{tabular}{|l|} 
High Gas \\
Concentration \\
Residue \\
\end{tabular} & $\begin{array}{l}\text { measure sensitivity after } \\
\text { exposure }\end{array}$ & Ongoing \\
\hline Dust & filter clogging & Ongoing \\
\hline Poisoning Test & 40 min of HMDSO & Ongoing \\
\hline Cross-Sensitivity & $\begin{array}{l}50 \% \mathrm{LEL} \text { of } \mathrm{H} 2, \mathrm{C} 2 \mathrm{H} 4 \\
\text { (ethene), } \mathrm{C} 3 \mathrm{H} 8 \\
\text { (propane), } \mathrm{C} 6 \mathrm{H} 14 \\
\text { (hexane), } \mathrm{C} 8 \mathrm{H} 18 \\
\text { (octane), } \mathrm{C} 9 \mathrm{H} 2 \mathrm{O} \\
\text { (nonane), } \mathrm{C} 2 \mathrm{H} 6 \mathrm{O} \\
\text { (ethanol) }\end{array}$ & Ongoing \\
\hline Effects of Oxigen & $\begin{array}{l}50 \% \text { LEL methane + O2 } \\
\text { levels at } 12 \ldots 21 \% \text { vol. }\end{array}$ & Ongoing \\
\hline Resolution & better than $1 \%$ LEL & Passed \\
\hline $\begin{array}{l}\text { Slow Increase of } \\
\text { Gas Volume }\end{array}$ & $\begin{array}{l}\text { increase of } 0.1 \% \text { LEL per } \\
15 \text { min up to } 6 \% \text { LEL }\end{array}$ & Ongoing \\
\hline $\begin{array}{l}\text { Effects of other } \\
\text { Gases } \\
\end{array}$ & $\mathrm{NO}, \mathrm{SO} 2$, & Ongoing \\
\hline \begin{tabular}{|l|} 
Sensitivity \\
\end{tabular} & $>5 \mathrm{mV} / \% \mathrm{vol}$ & Passed \\
\hline $\begin{array}{l}\text { Storage in High } \\
\text { Humidity }\end{array}$ & $\begin{array}{l}60^{\circ} \mathrm{C}, 95 \% \mathrm{RH} \text { for } 6 \\
\text { months }\end{array}$ & Ongoing \\
\hline $\begin{array}{l}\text { Storage at Low } \\
\text { Temperature }\end{array}$ & $-10^{\circ} \mathrm{C}$ for 6 months & Ongoing \\
\hline \begin{tabular}{|l|} 
Storage at High \\
Temperature \\
\end{tabular} & $60^{\circ} \mathrm{C}$ for 6 months & Ongoing \\
\hline
\end{tabular}

The above tests are extracted from international standards $[4 \ldots 10]$.

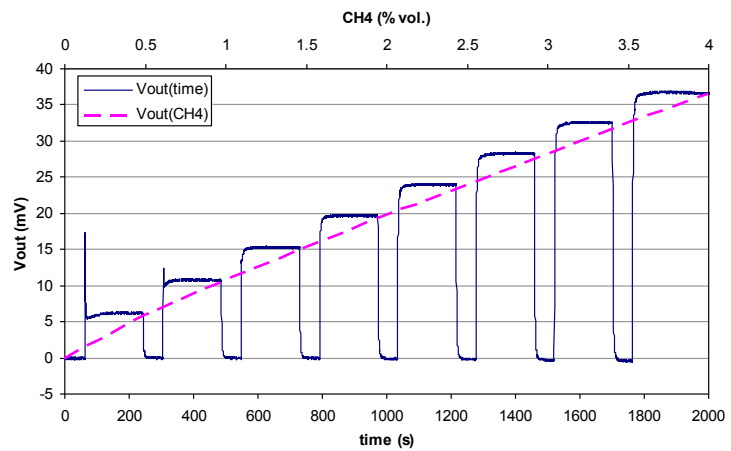

Fig. 6. Subsequent methane injections of increasing concentrations from $0.5 \%$ to $4.0 \%$ volume in synthetic air demonstrate the fast response time, excellent stability and linearity of the MEMS pellistor.
Our MEMS device will undergo all tests before product release scheduled for the second half of 2012. Current measurement results are highly promising as demonstrated in the following graph in Fig. 6.

In conclusion, significant progress has been made towards improving the mechanical robustness, lowering the power consumption, and reducing the overall size of the device as shown in the radar chart of Fig. 7.

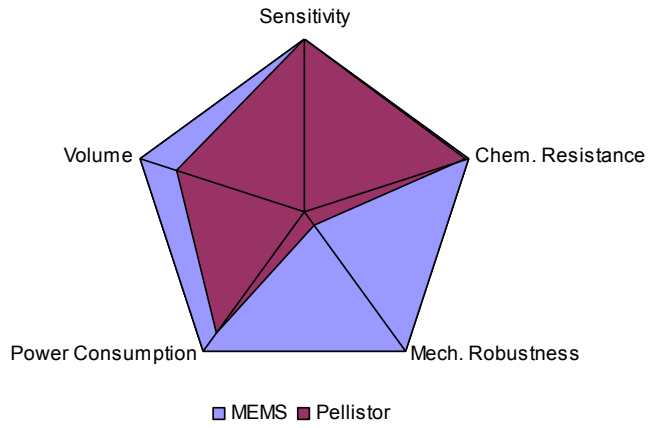

Fig. 7. Radar chart comparing sensitivity, chemical resistance, mechanical robustness, power consumption and volume of the new MEMS device with the pellistor. Axes are in logarithmic scales.

\section{References}

[1] http://www.e2v.com/products-andservices/instrumentation-solutions/gassensors/our-gas-sensor-technology/pellistorscatalytic-bead/

[2] http://www.e2v.com/e2v/assets/File/sensors_data sheets/Pellistors/pellistor_intro.pdf

[3] "Micromachined metal oxide gas sensors: opportunities to improve sensor performance Review Article" Isolde Simon, Nicolae Bârsan, Michael Bauer, Udo Weimar, Sensors and Actuators B: Chemical, Volume 73, Issue 1, 25 February 2001, Pages 1-26

[4] "Explosive atmospheres. Equipment. General requirements" EN 60079-0, 2009

[5] "Explosive atmospheres. Equipment protection by flameproof enclosures, d", EN 60079-1, 2007

[6] "Standard of Explosion-Proof and Dust-IgnitionProof Electrical Equipment for Use in Hazardous (Classified) Locations", UL 1203, $4^{\text {th }}$ Edition

[7] "Electrical Apparatus for the detection and Measurement of Flammable Gases", EN 617794, 2000

[8] "Coal mine carrier catalytic element for Methane detection", AQ 6202, 2006

[9] Electrical Apparatus for the Detection of Combustible Gases in Domestic Premises. Test Methods and Requirements", EN 50194, 2000

[10] "Parts 1 to 6 relating to the Domestic Appliances Approvals in China", GB15322, 2003 